NUMERICAL GAS-DYNAMIC INVESTIGATION OF THE WHIMPER MODEL FOR THE FORMATION OF PLANETARY NEBULAE

\author{
C.R. Purton \\ Dominion Radio Astrophysical Observatory, Penticton, B.C., \\ Canada
}

The suggestion that the shells of planetary nebulae may be formed at the interface between two stellar winds of different velocity ("Not with a bang, but a whimper") (Kwok, Purton and FitzGerald, 1978, Astrophys. J. 219, L 125) is investigated using the 'beam scheme' (Sanders and Prendergast, 1974, Astrophys. J. 188, 489) adapted to a system with spherical symmetry. Initial conditions include the remnant wind from the red giant phase $\left(\dot{M}=10^{-5} \mathrm{M}^{-1}, \mathrm{~V}=10 \mathrm{~km} \mathrm{~s}^{-1}\right)$ and a highspeed wind from the hot nucleus ( $\dot{m}=10^{-6^{\circ}} / 10^{-7} \mathrm{M} \mathrm{y}^{-1}$ (two tests), $\left.\mathrm{v}=10^{3} \mathrm{~km} \mathrm{~s}^{-1}\right)$. The collision began at a radial distance of $200 \mathrm{AU}$. A third test at $50 \mathrm{AU}$ indicated that the end result was insensitive to the details of the transition from one mass-loss mechanism to the other.

The shell formed at the interface increased in radius and mass: after several thousand years it had the characteristics of a conventional idealised planetary nebula. Calculated expansion velocities were in the range observed, and increased slowly with time. Temperatures of $2.5 \times 10^{7} \mathrm{~K}$ were found for the gas inside the she11, producing weak but observable (at $1 \mathrm{kpc}$ ) X-ray emission. $\lambda 4686$ emission produced by collisional ionization was found to be as strong as $\mathrm{H}_{\beta}$ emission at the later epochs. The radio spectrum at $\mathrm{T}=100 \mathrm{y}$ resembled $\mathrm{Hb} 12$, at $\mathrm{T}=500 \mathrm{y}$ a conventional 'optically thick' planetary, and after a few thousand years became optically thin.

Stellar mass loss, both components, clearly have a profound effect on the dynamics of a planetary nebula. It may not be necessary to invoke any sudden ejection for the formation of a planetary nebula; it may be the natural consequence of ordinary mass-loss processes in the two stages of the star's evolution.

ISAACMAN: The model predicts the presence of $10^{4} \mathrm{~K}$ gas with a density of about $10^{3} \mathrm{~cm}^{-3}$ outside the shell. This seems inconsistent with observations of $\mathrm{H}_{2}$ in several $\mathrm{PN}$, as $\mathrm{H}_{2}$ is presumably shock-excited by the expanding she11 but dissociates at a few thousand $\mathrm{K}$.

PURTON: The model assumes the gas to be ionized. A more realistic approach would be to follow the ionization front through the gas. 
TARTER: I believe that $\mathrm{X}$-ray line cooling will dominate bremsstrahlung at temperatures of $10^{6} \mathrm{~K}$. The diffuse emission in $\mathrm{X}$-rays could have an observable effect on high ionization trace species such as Fe XIV. DOPITA: The enhancement of X-ray cooling of the hot gas in nonequilibrium ionization conditions cannot be neglected. The large ionization time-scale of hydrogen- and helium-like ions ensures that a large amount of collisional excitation of their lines occurs. This raises the emissivity to about 30 times the equilibrium value.

\section{HBV 475 AS A CANDIDATE PROTO-PN}

S. Tamura

Astronomical Institute, Tohoku University, Sendai, Japan

The symbiotic nature of this object has been investigated by optical spectroscopy and photometry in the near infrared region. There are three components, which are (i) the ionized expanding envelope consisting of two layers of low and high excitation species, ( $i$ ) the late type component corresponding to a blackbody of $2500 \mathrm{~K}$ and indicated by TiO absorption band, (iii) the hot remnant star whose temperature is estimated indirectly as $150000 \mathrm{~K}$ or more.

Recently a high dispersion spectrum with self scanning diode array detector was obtained. If we assume circular motions in the Galactic plane, we can estimate the distance as $9 \mathrm{kpc}$ with the aid of the radial velocity. In this spectrum, we can also clearly see Fe I absorption lines $(\lambda 4325.8,4383.6,4404.8)$ shifted to the violet by about $10 \AA$ $\left(=-750 \mathrm{~km} \mathrm{~s}^{-1}\right)$ as well as broad $\mathrm{H \gamma}$ and (0 III) $\lambda 4363$ emission lines. In order to interpret these absorption lines, a single star hypothesis may be preferable.

On the basis of IR photometry and radio data, the dimensions of both the ionized expanding envelope and the late type component can be estimated. It should be considered that the late type component is extremely small and perhaps is embedded in the ionized expanding envelope.

To explain the observed data, a schematic model is proposed. (Papers will appear in Pub1. Astron. Soc. Japan). 